Quim. Nova, Vol. 36, No. 3, 462-467, 2013

\title{
INVENTÁRIO DE AGROTÓXICOS E RISCO DE CONTAMINAÇÃO QUÍMICA DOS RECURSOS HÍDRICOS NO SEMIÁRIDO CEARENSE
}

\author{
Allyne F. Gama, André H. B. de Oliveira e Rivelino M. Cavalcante* \\ Instituto de Ciências do Mar, Universidade Federal do Ceará, Av. Abolição, 3207, 60165-081 Fortaleza - CE, Brasil
}

Recebido em 30/3/12; aceito em 24/9/12; publicado na web em 18/2/13

\begin{abstract}
INVENTORY OF PESTICIDES AND RISK OF CHEMICAL CONTAMINATION OF HYDRIC RESOURCES IN THE SEMIARID
CEARENSE. The first diagnosis of risk of environmental contamination was conducted for an area of intense agricultural activity in the semiarid cearense (Baixo Jaguaribe and Litoral de Aracati area). We verified the use of 201 rural products (pesticides) in the region, comprising 151 active ingredients. Regarding the active ingredients evaluated, $15.9 \%$ were associated with sediment and $29.8 \%$ were dissolved in surface water. The study showed that $13.2 \%$ to $36.4 \%$ of the active ingredients evaluated were classified as potential contaminants of groundwater resources. The study also revealed the most abundant pesticides and which environmental compartment must be evaluated.
\end{abstract}

Keywords: risk assessment; semiarid cearense; pollution.

\section{INTRODUÇÃO}

A crescente demanda por alimentos tornou-se um problema de ordem global enfrentado por muitas nações. Nesse contexto, a agricultura moderna passou a utilizar de diferentes técnicas e insumos a fim de minimizar as perdas na produção. Entretanto, o uso indiscriminado desses diferentes insumos (agrotóxicos e fertilizantes) pode acarretar diversos prejuízos ambientais. ${ }^{1,2}$

No Brasil, a contaminação dos recursos hídricos pode ser potencializada, considerando que os dados gerados no estudo do Monitoramento do Mercado de Agrotóxicos, realizado pela Agência Nacional de Vigilância Sanitária - ANVISA, apontam maior ritmo de expansão no mercado brasileiro de consumo de agrotóxicos do mundo. O mercado brasileiro cresceu, aproximadamente, $176 \%$ na última década, ou seja, quatro vezes mais do que a média mundial. ${ }^{3}$

Há muito tempo, diversas políticas foram implementadas a fim de diminuir as mazelas socioeconômicas produzidas pela escassez de recursos hídricos no estado do Ceará. A partir do fim da década de 1970 tiveram início vários programas de irrigação no estado do Ceará no intuito de produzir alimentos, gerar empregos em atividades rurais e urbano-rurais, aumentar e melhorar a distribuição de renda, criando condições para a conquista da cidadania, através de setores de produção ligados ao campo. ${ }^{4}$

Historicamente os projetos de irrigações mais promissores são os perímetros irrigados instalados na Bacia Hidrográfica do Rio Jaguaribe, devido principalmente à disponibilidade de água e solo favorável, em especial os da Chapada do Apodi. ${ }^{5}$ Nos últimos 5 anos ocorreu um aumento de quatro a cinco vezes na área cultivada somente no setor do agronegócio, ${ }^{4}$ composta por produtores e empresas multinacionais do setor da fruticultura, para a exportação e mercado nacional. Levando-se em conta que o modelo de produção agrícola brasileiro se baseia na utilização de defensivos agrícolas para compensar problemas do processo produtivo, ${ }^{2,5,6} \mathrm{O}$ aumento na área cultivada foi acompanhado pelo aumento do uso de agrotóxicos na região.

Em áreas agrícolas a principal preocupação é com os recursos hídricos e especialmente para o semiárido nordestino essa preocupação é ainda maior em virtude do déficit hídrico dessa região.

\footnotetext{
*e-mail: rivelino@ufc.br
}

Desta forma, o conhecimento a respeito da qualidade dos recursos hídricos é fundamental nas regiões agrícolas, sendo que o principal modo de avaliar o impacto do setor e promover políticas voltadas à saúde e meio ambiente é através de programa de monitoramento da qualidade das águas. ${ }^{7}$ Entretanto, por se tratar de áreas amplas, lançamento de uma grande diversidade de agrotóxicos e dificuldade em laboratórios de análise e recursos humanos, programas de monitoramento apresentam um alto custo e podem não ser eficientes. Assim, estudos da determinação do perfil de agrotóxicos regional acompanhado do uso de modelos de avaliações de riscos são atualmente as principais ferramentas utilizadas em grandes áreas agrícolas no Brasil. ${ }^{7-15}$

Diante do fato que as propriedades físico-químicas dos agrotóxicos são governantes na adsorção pelos constituintes do solo (matéria orgânica, conteúdo argiloso e outros minerais), ou degradação por micro-organismos, bem como volatilização ou mobilidade (lixiviação), os modelos de avaliação preconizam quanto ao risco da contaminação por agrotóxico em águas subterrâneas e superficiais de forma simples e eficiente..$^{1,16-18}$ Esses dados podem ser utilizados em políticas agrícolas, por meio de medidas de banir agrotóxicos de alto risco ou de programas de conscientização rural, contribuindo também na elaboração de um planejamento para estudos de monitoramentos de pesticidas em água na região, reduzindo custo e tempo para o desenvolvimento. ${ }^{14}$

O desenvolvimento de inventários ambientais ou de fonte e emissão são frequentemente técnicas e metodologias usadas na determinação de impacto ambiental e como parte do procedimento de revisão, o qual precede a implementação de um sistema de gerenciamento ambiental. ${ }^{19}$ Em termos gerais, um inventário é uma lista detalhada dos produtos investigados lançados para o ambiente, ${ }^{19} \mathrm{a}$ fim de se obter informações que auxiliem no nível de contaminação ambiental, padrão de concentração do poluente, efeito causado pelo poluente no ambiente, compreensão a respeito do transporte e destino, bem como consequências socioeconômicas, risco à saúde humana e à qualidade ambiental. ${ }^{19,20}$

Desta forma, a primeira etapa de um estudo sobre a contaminação dos recursos hídricos por agrotóxicos deve ser um levantamento a respeito do lançamento dos princípios ativos na região estudada, características edafo-climáticas e das propriedades físico-químicas dos princípios ativos, permitindo o diagnóstico da contaminação 
ambiental. ${ }^{21}$ Com isto, os principais objetivos do presente estudo foram elaborar um inventário dos agrotóxicos utilizados nas microrregiões do Baixo Jaguaribe e Litoral de Aracati e, a partir dessa informação, produzir um diagnóstico do risco de contaminação dos recursos hídricos.

\section{PARTE EXPERIMENTAL}

\section{Área de estudo}

As microrregiões do Baixo Jaguaribe e Litoral de Aracati estão localizadas a leste do estado do Ceará (Figura 1S, material suplementar), são constituídas por 14 municípios, com uma área territorial de $12.098,545 \mathrm{~km}^{2}$ e população próxima de 421.249 habitantes. ${ }^{22}$

A Bacia Hidrográfica do rio Jaguaribe possui $80.547 \mathrm{~km}^{2}$, distribuída em 5 sub-bacias, representando aproximadamente 55\% do estado do Ceará, abrange 3.655 açudes e 81 municípios $(29,7 \%$ população cearense). Na sub-bacia do baixo Jaguaribe, estão inseridos os maiores complexos de irrigação do estado do Ceará nas cidades de Russas, Morada Nova, Limoeiro do Norte e Quixeré, composta por produtores agrícolas de pequeno a grande porte, bem como empresas multinacionais do setor da fruticultura, para a exportação e mercado nacional. O principal uso da água pela população dessa região é através de poços artesianos e por meio de pontos de captação, sendo importante salientar que o abastecimento de água da Região Metropolitana de Fortaleza é a partir da captação hídrica oriunda dessa bacia por meio do Canal do Trabalhador, tornando esta bacia uma das mais importantes em termos socioambiental e econômico do estado do Ceará. ${ }^{4}$

Os dados a respeito do tipo de solo e as condições edafo-climáticas da região foram obtidos a partir de banco de dados de órgãos do estado do Ceará. ${ }^{23,24}$ A Superintendência Estadual do Meio Ambiente (SEMACE) é o órgão responsável pelo registro dos agrotóxicos comercializados no estado do Ceará e tem um banco de dados on line (http://www.semace.ce.gov.br/florestal/agrotoxicos/ consulta-de-agrotoxicos-2/), para consulta pública dos "Produtos Cadastrados para o Comércio no Interior do Estado do Ceará", que é atualizado mensalmente. Esse cadastro é pioneiro entre os órgãos ambientais, auxiliando em ações de fiscalização. Segundo esse cadastro, é permitida a venda e administração de mais de 850 formulações produzidas por 78 empresas, os quais podem ser vendidos por mais de 250 comércios em todo o estado do Ceará. Somente nas microrregiões do Baixo Jaguaribe e Litoral de Aracati, localizadas nas bacias hidrográficas do baixo Jaguaribe e em uma parte da Bacia do Banabuiú, são encontrados 26 estabelecimentos comerciais que comercializam agrotóxicos. ${ }^{25}$

Os agrotóxicos para o estudo foram selecionados a partir do banco de dados da SEMACE, que são vendidos nos comércios especializados nos municípios das microrregiões do Baixo Jaguaribe e Litoral de Aracati, bem como através de dados coletados nas federações dos perímetros de irrigação (AUDIPIMN, DISTAR e FAPIJA),${ }^{4}$ médio e pequenos agricultores da área de estudo. Foi consultado também o banco de dados do sistema AGROFIT on line do Ministério da Agricultura (http://extranet.agricultura.gov. br/agrofit_cons/principal_agrofit_cons) quanto a detalhes a respeito dos princípios ativos dos agrotóxicos estudados e classificação toxicológica e ambiental. O AGROFIT on line é uma ferramenta de consulta pública, composta por um banco de dados de todos os produtos agrotóxicos e afins registrados no Ministério da Agricultura, Pecuária e Abastecimento, com informações do Ministério da Saúde (ANVISA) e do Ministério do Meio Ambiente (IBAMA). Permite vários tipos de pesquisas para o controle de pragas na agricultura brasileira, fornecendo informações também das marcas comerciais, culturas, ingredientes ativos, classificação toxicológica e classificação ambiental.

\section{Caracterização e propriedades físico-químicas dos agrotóxicos investigados}

O conhecimento das propriedades físico-químicas dos agrotóxicos, bem como sua estrutura e massa molecular são fundamentais para realização de um estudo sobre a dinâmica no ambiente. Para elaborar o diagnóstico do risco de contaminação dos recursos hídricos, pelos princípios ativos utilizados na região de estudo, foram utilizados os parâmetros: solubilidade em água $(\mathrm{S})$, coeficiente de adsorção à matéria orgânica do solo $\left(\mathrm{K}_{\mathrm{oc}}\right)$, pressão de vapor $\left(\mathrm{P}_{\mathrm{v}}\right)$, constante da Lei de Henry $\left(\mathrm{K}_{\mathrm{h}}\right)$, produto da constante de dissociação ácida do composto $\left(\mathrm{P}_{\mathrm{ka}}\right)$, tempo de meia-vida no solo $\left(\mathrm{DT}_{50 \text { solo }}\right)$ e na água $\left(\mathrm{DT}_{50 \text { água }}\right)$, retirados do global availability of information on agrochemicals. ${ }^{26}$

\section{Diagnóstico do risco de contaminação dos recursos hídricos}

O risco de contaminação de águas superficiais foi preconizado segundo o modelo proposto por Goss, que utiliza como parâmetros: constante de adsorção à matéria orgânica do solo $\left(\mathrm{K}_{\mathrm{oc}}\right)$; tempo de meia-vida no solo $\left(\mathrm{DT}_{50 \text { solo }}\right.$ ) e solubilidade em água a $25{ }^{\circ} \mathrm{C}$ dos princípios ativos investigados. ${ }^{9,27} \mathrm{De}$ acordo com o modelo de Goss, os contaminantes nos compartimento hídricos superficiais são transportados dissolvidos em água ou associados ao sedimento em suspensão. Os princípios ativos investigados seguem critérios de classificação em alto, médio ou baixo potencial de contaminação associado ao sedimento ou dissolvido em água, conforme síntese na Figura 2S, material suplementar. Princípios ativos que não se enquadram em nenhum dos grupos citados são considerados com potencial médio de transporte (MPTDA / MPTAS). ${ }^{9}$

O risco de contaminação de águas subterrâneas foi preconizado de acordo com os critérios da EPA (Agência de Proteção Ambiental Americana) e Índice de vulnerabilidade de águas subterrâneas (Groundwater Ubiquity Score - GUS), conforme estudos bem sucedidos anteriormente..$^{7,10,11,14}$

Os critérios de screening, sugeridos pela EPA, utilizam as propriedades físico-químicas dos princípios ativos investigados, bem como uma faixa característica para cada um deles, conforme apresentadas na Tabela 1.

Tabela 1. Propriedades físico-químicas utilizadas nos critérios de avaliação da EPA

\begin{tabular}{lc}
\hline Parâmetros dos pesticidas & Faixa \\
\hline Solubilidade em água $(\mathrm{mg} / \mathrm{L})$ & $>30$ \\
Coeficiente de adsorção à matéria orgânica $-\mathrm{K}_{\mathrm{OC}}\left(\mathrm{mL} \mathrm{g}^{-1}\right)$ & $<300-500$ \\
Constante da Lei de Henry $-\mathrm{K}_{\mathrm{H}}\left(\mathrm{Pa} \mathrm{m}^{3} \mathrm{~mol}^{-1}\right)$ & $<10-2$ \\
Meia-vida no solo $\left(\mathrm{DT}_{50 \text { solo }}\right)($ dias $)$ & $>14-21$ \\
Meia-vida na água $\left(\mathrm{DT}_{50 \text { agua }}\right)($ dias $)$ & $>175$ \\
\hline
\end{tabular}

O cálculo do índice GUS para um princípio ativo investigado considera os valores do $\mathrm{K}_{\mathrm{oc}}$ e da meia-vida da substância no solo, os quais serão aplicados na equação: GUS $=\log \left(\mathrm{t}_{1 / 2}\right.$ solo $)$ x $(4-\log$ $\left.\mathrm{K}_{\mathrm{oc}}\right) .{ }^{28}$ Segundo o modelo, os princípios ativos investigados são classificados conforme sua tendência a sofrer lixiviação para o subterrâneo, de acordo com os seguintes intervalos: GUS $<1,8$ (o princípio ativo em questão não sofre lixiviação e é classificado como não contaminante (NC) de água subterrânea); 1,8 < GUS $<2,8$ (faixa de transição (T)) e GUS $>2,8$ (o princípio ativo em questão é classificado como potencial contaminante (PC) de água subterrânea). 


\section{RESULTADOS E DISCUSSÃO}

\section{Inventário}

Os dados gerais do inventário de agrotóxicos utilizados na região de estudo estão sumarizados na Tabela $1 \mathrm{~S}$, material suplementar. Através dos dados foi verificado o uso de 201 produtos agrícolas produzidos por 21 empresas. Dos 201 produtos agrícolas encontrados na região, 3 não apresentam registro no sistema AGROFIT on line e nem no cadastro on line da SEMACE, e 1 apesar de fazer parte do sistema AGROFIT on line não tem o princípio ativo definido. Os agrotóxicos sem registro podem ser devidos: a necessidades emergenciais; produtos não autorizados para venda como defensivos agrícolas ou contrabando, em casos menos comuns. Foram verificados 148 princípios ativos com classe química definida e 2 compostos denominados de óleos mineral e vegetal, os quais podem estar presentes de forma unificada ou através de misturas contento dois ou até três princípios ativos por produto agrícola (Tabela $1 \mathrm{~S}$, material complementar).

O grande número de agrotóxicos administrados nessa região é reflexo do aumento da área de plantio, impulsionado pelo crescimento no agronegócio e pela melhoria e incentivos ao médio e pequeno agricultor e, principalmente, pela isenção de impostos. A análise dos dados do inventário de agrotóxicos mostrou uma nova realidade no semiárido nordestino, uma vez que nas décadas de 80 e 90 poucas lojas de produtos agrícolas eram encontradas na região e o uso de agrotóxicos era reduzido e limitado a poucas marcas e princípios ativos.

Comparando com levantamentos nas principais regiões agrícolas do país (Tabela 2), a diversificação de produtos agrícolas administrados na região estudada, bem como dos princípios ativos, é bem maior quando comparada com estudos nas regiões Sul e Sudeste. Isso torna a região dos perímetros de irrigação dos municípios de Russas, Morada Nova, Limoeiro do Norte e Quixeré um dos maiores e mais diversificados no uso de agrotóxicos no país. Segundo Dores e De-Lamonica-Freire, ${ }^{9}$ a tendência nacional é o uso de herbicida seguido de inseticida, entretanto na região de estudo verificou-se a predominância do uso de fungicida seguido de herbicida e inseticida, e não foi verificado padrão semelhante com outras regiões (Tabela 2).

As culturas predominantes com mais de 1000 ha em área de plantio são arroz, banana, milho e feijão. ${ }^{4}$ Analisando os dados do inventário, especialmente os produtos agrícolas administrados na região de estudo, entre 60 e $70 \%$ podem ser aplicáveis nas culturas do milho ou feijão e 32,3\% em ambos, além de outras culturas. Esse dado é interessante, uma vez que as culturas do milho e feijão são as principais dos pequenos agricultores ou da agricultura familiar, bem como dos grandes agricultores no semiárido cearense. Como essas culturas são populares e não necessitam grandes recursos na produção, o comércio direcionou a venda dos produtos em função das culturas predominantes. Esse direcionamento pode ser devido à procura, reflexo da diminuição do preço desses "venenos" e políticas de divulgação maciças promovidas pelas empresas fabricantes. A popularização dos agrotóxicos em países em desenvolvimento é crescente e um dos maiores problemas apontados pela mídia internacional, assim pequenos agricultores estão usando esse artifício na compensação de perdas na agricultura, abandonando práticas alternativas do passado, as quais não apresentam resultados tão rápidos, quando comparados com o uso de agrotóxicos.

Dos 198 produtos agrícolas administrados na região de estudo quanto à classificação toxicológica, $48 \%$ pertencem às classes I e II (extremamente e altamente tóxicos, respectivamente) (Figura 1). Quanto à classificação ambiental, mais de $60 \%$ pertencem às classes I e II (Produto Altamente Perigoso e Muito Perigoso ao Meio Ambiente, respectivamente). Esse padrão de perfil toxicológico e ambiental dos agrotóxicos estudado na região é semelhante aos encontrados em levantamentos recentes realizados em Minas Gerais, sendo importante salientar que o autor já chamava atenção para a necessidade de estudos e a revisão sobre a utilização destes agrotóxicos nas atividades agrícolas da região. ${ }^{7}$ Esses dados mostram também que a adoção por agrotóxicos mais "potentes" (mais tóxicos e de maior perigo ambiental) parecer ser predominante e crescente, que práticas alternativas estão sendo abandonadas e políticas agrícolas de conscientização não estão funcionando ou o levantamento e discussão do problema pela mídia e setores não está surtindo nenhum efeito.

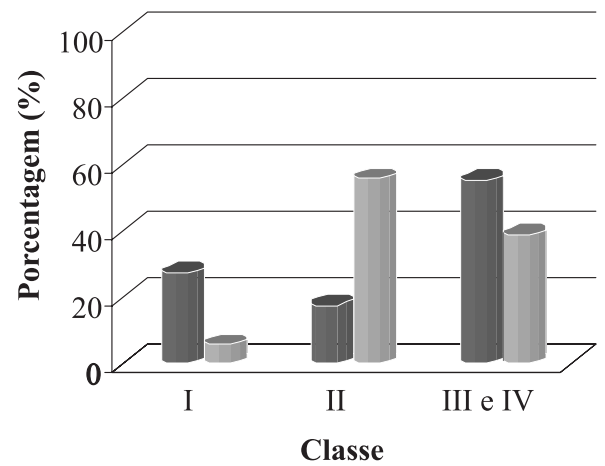

- Toxicológica

$\square$ Ambiental

Figura 1. Percentagem das classes tóxicas e ambientais

Os princípios ativos abamectina, alfa-cipermetrina, ametrina, atrazina, clorotalonil, clorpirifós, diurom, glifosato, imidacloprido e tiofanato-metílico apresentam-se como os mais frequentes nos produtos agrícolas utilizados na região estudada (Figura 2). Todos são das classes toxicológica e ambiental I e II, demonstrando que a adoção por agrotóxicos mais perigosos é a principal alternativa na região.

\section{Diagnóstico}

O resultado da forma de transporte e do diagnóstico de risco de contaminação dos recursos hídricos pelos princípios ativos

Tabela 2. Dados dos agrotóxicos na região de estudo e outros estados

\begin{tabular}{|c|c|c|c|}
\hline Local & $\begin{array}{l}\text { Produto agrícola/ } \\
\text { princípio ativo }\end{array}$ & Cultura predominante & Predominância \\
\hline Rio Grande e Santa Vitória do Palmar - RS ${ }^{11}$ & 27 & Arroz, cebola e tomate & Herbicidas \\
\hline Primavera do Leste $-\mathrm{MT}^{9}$ & 30 & Soja, milho e arroz & Herbicidas $>$ inseticidas $>$ fungicidas \\
\hline Petrolina - PE e Juazeiro - BA ${ }^{29}$ & 39 & Manga e uva & Inseticidas $>$ fungicidas $>$ herbicidas \\
\hline Região de Guaíra -SP18 & 19 & Feijão, soja, milho e tomate & Fungicidas $>$ inseticidas $>$ herbicidas \\
\hline Rio Paranaíba, São Gotardo e Ibiá - MG ${ }^{7}$ & $59 / 62$ & $\begin{array}{l}\text { Café, milho, soja, feijão, batata-inglesa, beter- } \\
\text { raba, cebola, cenoura, alho, trigo e cana-de-açúcar }\end{array}$ & Inseticidas $>$ fungicidas $>$ herbicidas \\
\hline Baixo Jaguaribe e Litoral de Aracati -CE & $201 / 151$ & $\begin{array}{l}\text { Arroz, banana, capim de corte, mamão, melancia, } \\
\text { melão, milho, feijão, soja e sorgo }\end{array}$ & Fungicidas $>$ herbicidas $>$ inseticidas \\
\hline
\end{tabular}




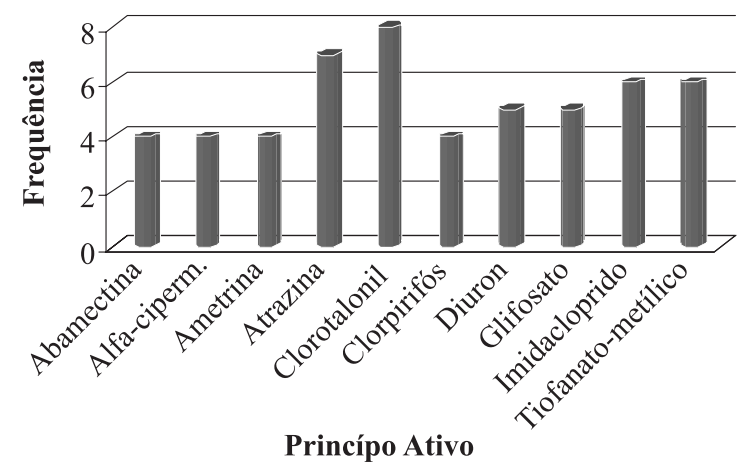

Figura 2. Princípios ativos mais utilizados na região de estudo (frequência por produto agrícola)

encontrados na região de estudo estão apresentados na Tabela $2 \mathrm{~S}$, material complementar.

Conforme os dados da Tabela 2S, sintetizados na Figura 3, pode-se verificar que dos princípios ativos avaliados pelo modelo Goss 43,7\% apresentam potencial médio de contaminação de água superficial com transporte associado ao sedimento, seguido de $27,8 \% \mathrm{com}$ potencial baixo e de $15,9 \%$ em alto. Em contrapartida, o transporte de contaminantes dissolvidos em água seguiu uma tendência diferenciada, os princípios ativos investigados foram classificados em 39,1\% com potencial médio, seguido de $29,8 \%$ em alto e $19,2 \%$ em baixo (Figura 3). Em torno de 12,0\% dos princípios ativos investigados não foram definidos quanto a sua forma de transporte, e isso é em virtude da ausência de informações na literatura especializada.

- Associado ao sedimento - Dissolvido na Água

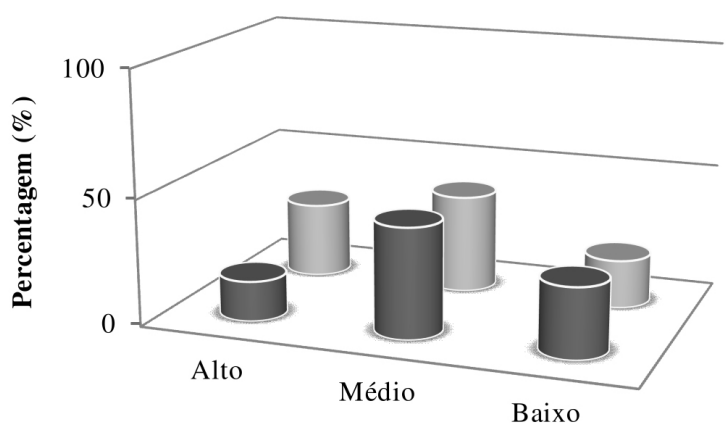

Classificação modelo Goos

Figura 3. Forma de transporte dos princípios ativos na região de estudo

O padrão de classificação dos princípios ativos avaliados, utilizando o modelo Goss, foi o mesmo verificado em Rio Paranaíba, São Gotardo e Ibiá-MG $\mathrm{MG}^{7}$ para ambas as formas de transporte. Entretanto, comparado com os princípios ativos estudados em Rio Grande e Santa Vitória do Palmar-RS ${ }^{11}$ somente apresentou o mesmo padrão de classificação para o transporte de contaminantes dissolvidos em água.

O risco de contaminação dos recursos hídricos subterrâneos, utilizando o Índice de GUS e os critérios da EPA estão resumidos na Figura 4. Como pode ser verificado, para o índice de GUS, 13,2\% se enquadram como contaminantes potenciais, $17,9 \%$ estão na faixa de transição, $56,3 \%$ são classificados como não contaminantes e $11,3 \%$ inconclusivos. Utilizando os critérios da EPA, observa-se que $36,4 \%$ são enquadrados como contaminantes potenciais, seguido de $34,4 \%$ como não contaminantes, $21,9 \%$ na faixa de transição e $6,0 \%$ inconclusivos. Já a análise considerando ambos os critérios (GUS e EPA) mostrou que 46,4\% são enquadrados como não contaminantes,

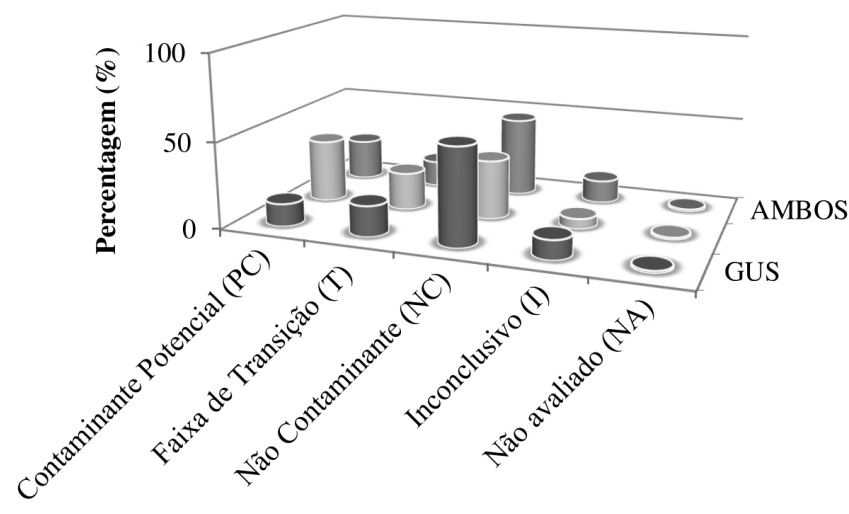

Critérios

Figura 4. Resultado do risco de contaminação das águas subterrâneas

seguindo por $23,8 \%$ como contaminantes potenciais, $15,2 \%$ na faixa de transição e 13,2\% inconclusivos. Em ambos os critérios, 1,3\% (por exemplo, óleo mineral e vegetal) não foram avaliados por não serem classificados como princípio ativo (Figura 4).

Para alguns princípios ativos, foi observada discordância entre os modelos GUS e EPA no diagnóstico de risco de contaminação para as águas subterrâneas. Apesar do modelo GUS apresentar boa consonância com estudos de mobilidade de agrotóxicos em solo brasileiros, ${ }^{31}$ a discordância entre GUS e EPA em alguns dos resultados pode ser justificada pelas especificações dos critérios adotados em cada modelo. O critério da EPA utiliza um número maior de propriedades em sua avaliação em relação ao modelo GUS. Princípios ativos classificados na faixa de transição ou de elevado potencial de risco de contaminação necessitam de estudos mais aprofundados usando modelos mais eficientes e conclusivos, tais como o Índice de LIX e AF (fator de atenuação), bem como avaliação da capacidade de adsorção, os quais utilizam o próprio solo, bem como suas características edáficas. ${ }^{8,30}$

O diagnóstico do risco de contaminação dos recursos hídricos subterrâneos, para os princípios ativos investigados, tem se mostrado reprodutivo com estudos realizados utilizando modelos de adsorção em diversos solos de áreas agricultáveis do país. Os princípios ativos clorpirifós, endossulfam, lambda-cialotrina, diuron e atrazina em diversos solos (por exemplo, Neossolo Quartzarênico; Latossolo Psamítico; Vertissolo Ebânico e Latossolo Vermelho) têm a mobilidade reduzida em virtude do conteúdo de matéria orgânica desses tipos de solo. ${ }^{31-33}$ Esse fato é reforçado por Barreto, ${ }^{34}$ que avaliou a mobilidade de atrazina em solo pobre em conteúdo de matéria orgânica do município de Tianguá-CE, encontrando em torno de 50\% das amostras de água subterrânea a presença desse princípio ativo. Assim, alguns princípios ativos apresentam uma maior tendência de mobilidade em regiões com solo contendo baixo conteúdo de matéria orgânica, ${ }^{27}$ apresentando maior risco de contaminação de águas subterrâneas, o que está conforme o presente diagnóstico de risco de contaminação na região do Baixo Jaguaribe e Litoral de Aracati.

Em contrapartida, tiametoxam e acefato apresentam considerável mobilidade em Latossolo ${ }^{35}$ e Argissolo Vermelho-Amarelo, ${ }^{36}$ respectivamente. Desta forma, assim como nos estudos de capacidade de adsorção do tiametoxam e acefato em solos, o presente diagnóstico também aponta um considerável risco de contaminação dos recursos hídricos subterrâneos. Filizola e colaboradores ${ }^{37}$ realizaram a avaliação do risco de contaminação por agrotóxicos em águas superficial e subterrânea na região de Guaíra-PR, e verificaram a presença de clorotalonil, captan, endosulfan e lambda-cialotrina, entre outros, em água superficial. Entretanto, esses princípios ativos não foram encontrados em amostras de água subterrânea, levando os autores a 
Tabela 3. Síntese dos agrotóxicos prioritários em estudos de monitoramento ambiental na região do Baixo Jaguaribe e Litoral de Aracati

Compartimento ambiental Princípios ativos prioritários em monitoramento

Água superficial Ametrina, Atrazina, Azoxistrobina, Benalaxil, Boscalida, Bromacila, Carbendazim, Ciproconazol, Ciromazina, Clomazona, Clorpirifós, Clotianidina, Difenoconazol, Dimetomorfe, Diurom, Epoxiconazol, Fenarimol, Fenitrotiona, Fipronil, Fludioxonil, Fluopicolide, Flutriafol, Fomesafem, Imazalil, Imazapique, Imazetapir, Imidacloprido, Iprodiona, Metalaxil-M, Metconazol, Metolacloro, Paclobutrazol, Parationa-metílica, Picloram, Pirimetanil, Procloraz, Propiconazol, Tebuconazol, Tepraloxidim, Tiabendazol, Tiacloprido, Tiametoxam, Tidiazurom, Tiodicarbe, Tiofanato-metílico.

Água subterrânea 2,4-D, Alacloro, Ametrina, Atrazina, Azoxistrobina, Bentazona, Boscalida, Bromacila, Carbaril, Carbendazim, Carbofurano, Ciproconazol, Ciromazina, Clomazona, Clotianidina, Cresoxim-metílico, Dimetomorfe, Diurom, Epoxiconazol, Etoxissulfurom, Fenarimol, Fipronil, Fluopicolide, Flutriafol, Fomesafem, Imazamoxi, Imazapique, Imazetapir, Imidacloprido, Metalaxil-M, Metamidofós, Metolacloro, Nicosulfurom, Paclobutrazol, Picloram, Pirimetanil, Propiconazol, S-metolacloro, Tebuconazol, Tembotriona, Tepraloxidim e Tiametoxam.

Solo

Acibenzolar-S-metílico, Alfa-cipermetrina, Bacillus thuringiensis, Beta-ciflutrina, Beta-cipermetrina, Buprofezina, Captana, Carbosulfano, Cartap, Cialofope-butílico, Clorfenapir, Clorfluazurom, Clorotalonil, Clorpirifós, Deltametrina, Diafentiurom, Diflubenzurom, Endossulfan, Enxofre, Esfenvalerato, Etofenproxi, Famoxadona, Fenamidona, Fenoxaprope-P-etílico, Fenpiroximato, Fenpropatrina, Fluazifope-P-butílico, Fluazinam, Flubendiamida, Folpete, Fosetil, Fosfeto de alumínio, Fosfeto de magnésio, Glifosato-sal de potássio, Hidróxido de cobre, Hidróxido de fentina, Imibenconazol, Indoxacarbe, Lambda-cialotrina, Lufenurom, Mancozebe, Metiram, Oxicloreto de cobre, Óxido de fembutatina, Permetrina, Piraflufem, Procimidona, Profenofós, Profoxidim, Quizalofope-P-etílico, Sulfluramida, Terbufós, Tiodicarbe, Tiram, Trifloxistrobina, Triflumizol, Trifluralina.

concluírem que esse comportamento é devido às propriedades físicas e químicas do solo, aliado às dos agrotóxicos, que favorecem a retenção pelo solo. Segundo o presente diagnóstico, esses princípios ativos não se apresentam como um potencial contaminante para os recursos hídricos subterrâneos, corroborando e fortalecendo mais ainda que o uso de modelos de avaliação de risco é ferramenta poderosa no monitoramento e avaliação de risco de contaminação por agrotóxicos.

\section{Princípios ativos prioritários em programas de monitoramentos}

Em virtude da grande quantidade de agrotóxicos utilizados na região de estudo e considerável extensão territorial, em termos de aplicação, um programa de monitoramento ou de impacto ambiental tomaria um tempo extenso, bem como implicaria em altos custos de execução. O diagnóstico do risco de contaminação desponta como uma ferramenta eficiente no auxílio de tomadas de decisões e estratégias nesse âmbito. Desta forma, na Tabela 3 encontra-se uma síntese dos agrotóxicos prioritários em estudos de monitoramento ambiental e os prováveis compartimentos ambientais, onde podem ser encontrados com maior probabilidade. Como pode ser verificado, 31,3\% dos princípios ativos utilizados na região do Baixo Jaguaribe e Litoral de Aracati apresentam grande possibilidade de se encontrarem nos recursos hídricos superficiais e 28,6\% nos recursos hídricos subterrâneos. Vale destacar que a grande maioria dos princípios ativos investigados na região $(39,5 \%)$ pode ser encontrada no solo. Essa informação é bastante relevante, uma vez que dependendo da estação do ano, esses princípios ativos podem sofrer lixiviação ou escoamento superficial. Através da lixiviação, podem ser transportados para os recursos hídricos subterrâneos. Já o escoamento superficial favorece a contaminação das águas superficiais, que pode ocorrer através do transporte dos princípios ativos adsorvidos às partículas do solo erodido ou em solução. ${ }^{38}$ Ambos os modos de transporte vão contribuir para a contaminação dos recursos hídricos da região.

\section{CONCLUSÃO}

O inventario de agrotóxico elaborado para a região do Baixo Jaguaribe e Litoral de Aracati mostrou que uma grande quantidade de agrotóxicos está sendo lançada na região do semiárido cearense. O perfil toxicológico e ambiental dos agrotóxicos lançados na região também é um fator preocupante, mostrando que a adoção de agrotóxicos mais "potentes" e perigosos parece ser predominante e crescente.

Os dados oriundos do diagnóstico do risco de contaminação por agrotóxicos na região se mostraram bastantes coerentes com os apresentados em estudos publicados sobre a mobilidade de agrotóxicos em solos brasileiros. As informações geradas neste estudo certamente servirão como alerta, podendo ser utilizadas como ferramentas norteadoras no auxílio de tomadas de decisões e elaborações de políticas de monitoramentos, avaliação de impactos e programas de gerenciamento rural. Nesse âmbito, a elaboração de mapa de sensibilidade ambiental, que forneceria informações a respeito de áreas sensíveis ao uso de determinados agrotóxicos, seria um avanço promissor na diminuição dos impactos agrícolas.

A informação gerada neste estudo certamente representa a primeira iniciativa para a avaliação da condição ambiental nessa região e poderá ser aproveitada como ações visando à sustentabilidade, manutenção do equilíbrio e qualidade ambiental de uma região tão importante e sensível como o semiárido brasileiro.

\section{MATERIAL SUPLEMENTAR}

Está disponível em http://quimicanova.sbq.org.br, na forma de arquivo pdf, com acesso livre.

\section{AGRADECIMENTOS}

Esse trabalho foi subsidiado pelo CNPq (Proc. 484171/2010-0) e FUNCAP (Proc. 150.01.00/09). Os autores agradecem também a Superintendência Estadual do Meio Ambiente (SEMACE), em especialmente ao setor de Diretoria Florestal (DIFLO) pela disponibilidade das informações.

\section{REFERÊNCIAS}

1. Sanches, S. M.; Silva, C. H. T. P.; Campos, S. X.; Vieira, E. M.; Pesticidas. Ecotoxicol. Meio Amb. 2003, 13, 53.

2. Veiga, M. M.; Ciência \& Saúde Coletiva 2007, 12, 145.

3. http://portal.anvisa.gov.br/wps/portal/anvisa/home, acessada em Janeiro 2013.

4. http://www.dnocs.gov.br, acessada em Janeiro 2013.

5. Amaral, C. R.; Dissertação de Mestrado, Universidade Federal do Ceará, Brasil, 2007.

6. Lara, W. H.; de Batista, G. C.; Quim. Nova 1992, 15, 161. 
7. Andrade, A. S.; Queiroz, V. T.; Lima, D. T.; Drumond, L. C. D.; Quim. Nova 2011, 34, 1129.

8. Spadotto, C. A.; Pesticidas: Rev. Ecotoxicol. Meio Amb. 2002, 12, 69.

9. Dores, E. F. G. de C.; De-Lamonica-Freire, E. M.; Quim. Nova 2001, $24,27$.

10. Lourencetti, C.; Ribeiro, M. L.; Santiago-Silva, M.; Spadotto, C. A.; Pesticidas:. Ecotoxicol. Meio Amb. 2005, 15, 1 .

11. Cabrera, L.; Costa, F. P.; Primel, E. G.; Quim. Nova 2008, 31, 1982.

12. Queiroz, S. C. N.; Ferracini, V. L.; Gomes, M. A. F.; Rosa, M. A.; Quim. Nova 2009, 32, 378.

13. Spadotto, C. A.; Gomes, M. A. F.; Luchini, L. C.; Andréa, M. M.; Embrapa Meio Ambiente: Jaguariuna 2004, 42, 12.

14. Primel, E. G.; Zanella, R.; Kurz, M. H. S.; Gonçalves, F. F.; Machado, S. O.; Marchenza, E.; Quim. Nova 2005, 28, 605.

15. Milhome, M. A. L.; Sousa, D. O. B.; Lima, F. A. F.; Nascimento, R. F.; Engenharia Sanitária Ambiental 2009, 14, 363.

16. Hantush, M. M.; Mariňo, M. A.; Islam, M. R.; J. Hydrology 2000, 227, 66

17. Paraíba, L. C.; Cerdeira, A. L.; Silva, E. F.; Martins, J. S.; Coutinho, H. L. C.; Chemosphere 2003, 53, 1087.

18. Spadotto, C. A.; Filizola, H.; Gomes, M. A.; Pesticidas: Rev. Ecotoxicol. Meio Amb. 2001, 11, 127.

19. Lewis, K. A.; Newbold, M. J.; Tzilivakis, J.; J. Environ Manage. 1999, $55,183$.

20. http://www.epa.gov/, acessada em Janeiro 2013.

21. Dores, E. F. G. de C.; De-Lamonica-Freire, E. M.; Pesticidas: $R$. Ecotoxicol. Meio Amb. 1999, 9, 1.

22. http://www.ibge.gov.br/home/, acessada em Janeiro 2013.
23. http://www.ipece.ce.gov.br, acessada em Janeiro 2013.

24. http://www.funceme.br, acessada em Agosto 2012.

25. http://www.semace.ce.gov.br, acessada em Agosto 2011.

26. http://sitem.herts.ac.uk/aeru/iupac/, acessada em Fevereiro 2012

27. Goss, D. W.; Weed Technol. 1992, 6, 701.

28. Gustafson, D. I.; Environ. Toxicol. Chem. 1989, 8, 339.

29. Ferracini, V. L.; Pessoa, M. C. Y. P.; Silva, A. S.; Spadotto, C. A.; Pesticidas: Rev. Ecotoxicol. Meio Amb. 2001, 11, 1.

30. Cohen, S. Z.; Wauchope, R. D.; Klein, A. W.; Eadsforth, C. V.; Graney, R.; Pure Appl. Chem. 1995, 67, 2109.

31. Laabs, V.; Amelung, W.; Pinto, A.; Zech, W.; J. Environ. Qual. 2000, 31, 256.

32. Matallo, M. B.; Luchini, L. C.; Gomes, M. A. F.; Spadotto, C. A.; Cerdeira, A. L.; Marin, G. C.; Pesticidas. R. Ecotoxicol. Meio Amb. 2003, 13, 83.

33. Dick, D. P.; Martinazzo, R.; Knicker, H.; Almeida, P. S. G.; Quim. Nova 2010, 33, 14.

34. Barreto, F. M. S.; Tese de Doutorado, Universidade Federal do Ceará, Brasil, 2006.

35. Oliveira, V. S. O.; Lima, J. M.; Carvalho, R. F.; Quim. Nova 2009, 32, 1432.

36. Marchetti, M.; Luchini, L. C.; Pesticidas: R. Ecotoxicol. Meio Amb. 2004, 14, 61.

37. Filizola, H. F.; Ferracini, V. L.; Sans, L. M. A.; Gomes, M. A. F.; Ferreira, C. J. A.; Pesq. Agrop. Bras. 2002, 37, 659.

38. Queiroz, G. M. P.; Silva, M. R.; Bianco, R. J. F.; Pinheiro, A.; Kaufmann, V.; Quim. Nova 2011, 34, 190. 